\title{
OLBP on DWT Segments for Effective Face Recognition
}

\author{
Srinivas Halvi \\ Department of Medical Electronics, \\ Dayananda Sagar College of \\ Engineering, VTU, Bangalore, \\ India.
}

\author{
K B Raja \\ Department of Electronics and \\ Communication Engineering, \\ University Visvesvaraya College of \\ Engineering, Bangalore University, \\ Bangalore, India.
}

\author{
Shanti Prasad M J \\ Department of Electronics and \\ Communication Engineering, \\ K.S. Institute of Technology, VTU, \\ Bangalore, India.
}

\begin{abstract}
The real time applications in modern technology use face recognition to authenticate human beings for secure transactions. In this paper, we propose OLBP on DWT Segments for Effective Face Recognition. The regular face image databases are deliberated to exam our technique and applied Discrete Wavelet Transform (DWT). The LL band is considered and resized for four Symmetrical Vertical Segments (SVS). The Overlap Local Binary Pattern (OLBP) is applied to each vertical segment. The four OLBP segments are flipped vertically and combined with corresponding original OLBP segments to compute average OLBP values. The final OLBP features are obtained by concatenation of four segmented features. The probe image features relate to features of the face image database by Euclidian Distance (ED) to calculate performance parameters. It is witnessed that, the results of our technique is enhanced than the present techniques.
\end{abstract}

Keywords: Biometrics, DWT, ED, Face Recognition, OLBP.

\section{INTRODUCTION}

Biometrics are approaches of identifying a person based on body parts and behavioral characteristics and are highly secure identification and personal authentication systems. It is a fast developing field with claims ranging from firmly opening one's computer to gaining admission into a nation, whereas the placement of large-scale biometric systems in both marketing and government applications has improved the public consciousness of this machinery. The biometrics for personal authentication is pretty stable and significantly precise than the current systems viz., passwords and PINs. The biometrics are the utmost protected and suitable confirmation instrument, which can't be hired, whipped, forgotten and falsifying is almost difficult.

A biometric scheme operates in two modes viz., (i) verification comprises a one-to-one competition that compares a request image with an enrolment image whose identity is being claimed and (ii) identification includes oneto-many matching that compares a request image with multiple images in the enrolment database. Biometrics are generally classified into physiological and behavioral Biometrics. A physiological trait such as a fingerprint, hand geometry, iris pattern or blood vessel shape on the rear of the eye is relatively stable. A behavioral trait is more of an individual's emotional, which varies over a period of time, and examples are signature, keystroke, gait, speech, etc.
The prevailing and low-cost computing systems have shaped vast attention in automatic handling of digital face images in a diversity of applications, comprising biometric validation, investigation, human-computer communication, and multimedia administration. Face recognition has several benefits over other biometric modalities, as images are captured at a distance and in a secret way with the help of surveillance cameras and mobile phones. Face recognition is a system accomplished to identify or verify a human being from an image or a video frame. The numerous approaches in facial authentication schemes work by equating designated facial features of query images with faces images in the database. The face is the maximum expressive part of the human physique and one can decide the qualities and characteristics of an individual. The difficulty of face finding is highly nonlinear as the face is strongly animated and is challenging to extract statistics in a strong and effective way. The challenges in face recognition are variations in face images due to age [1-3], image resolution [4,5], facial expressions [6], poses [7] and different lighting conditions [8] causes changes to face images resulting low performance of face recognition system. The Local Binary Pattern (LBP) method was introduced by Ojala et al., [9] and is used as a dominant texture descriptor. The LBP technique is useful in the face recognition method for controlled conditions of face images and declines the performance of the face recognition system with differences in illuminations, expressions, and poses. The fact that the human face is approximately symmetrical, hence Shui-Guang Tong et al., [10] proposed Multi-Mirror Local Binary Pattern (MMLBP) and eliminated the effect of interferences on the performance of the system using LBP.

Contributions: In this paper, OLBP on DWT Segments for Effective Face Recognition is proposed. The DWT is used and considered only LL band. The symmetrical vertical segmentation process is initiated on LL band to obtain four segments. The OLBP on each segment is applied and flipped. The average of the original and flipped OLBP segment are computed to extract early features. The last features are gained by concatenating early features. The ED is used in matching technique to calculate performance results.

The paper is prepared as follows: we briefly summarize the literature survey of existing techniques of face recognition in Section II. Our algorithm details are given in Section III, 
and Section IV presents investigational results. The last section comprises conclusion of this paper.

\section{LITERATURE SURVEY}

In this division, the study of current research work on face recognition exploitation numerous techniques are mentioned. Bin Xiao et al., [11] have projected a two-dimensional LBP technique, that use a window to sum the weighted prevalence range of the spin invariant constant LBP pattern couples to get the spatial discourse data. A two-stage classifier step is employed to attain associate degree correct classification by merging the forecasts on every 2D-LBP by lone determination. Chao Qi et al., [12] have used pseudo-3D model to section the face space into six facial look subregions. During this framework, the sub-regions and also the international facial look pictures use the aforethought LBP technique for feature extraction, so use two arrangements that are the support vector machine and Soft GHB with two sorts of feeling classification prototypes like basic feeling model and also the diacritical mark feeling model. Latha and Savita S Kudakunti [13] have preprocessed the face pictures of ORL and JAFEE databases by exploitation of Histogram Equalization (HE). The DWT is used on HE pictures to get four bands. The OLBP is used on LL band to get final options. The options of check pictures are equated with the options of test pictures exploitation using ED. Arti Mahore and Meenakshi Tripathi [14] have introduced a method to observe the existence of 3D mask-based face anti-spoofing exploitation frequency and texture-based feature descriptors. The method extracts LBP primarily based texture options of DWT pictures. The DWT is employed to divide original image into low and Highfrequency bands in frequency domain and also the mask lacks in high-frequency parts. The colour pictures are used rather than grey pictures as a result of chrominance half is absent in grey pictures. The experiment on $\mathrm{YCbCr}$ colour area is performed rather than $\mathrm{RGB}$ as a result of in $\mathrm{YCbCr}$, the luminousness and Chrominance half is separated. These distinct colour channels are then treated by exploitation DWT. The frames gained from DWT are then managed by LBP. The acquired LBP image is separated into nonoverlapping identical dimension slabs. Feature vector of every block is concatenated to make one feature vector for a picture. The resulting feature vector is served to classifier to express apart between actual and cover.

Jun-Gu Lee and Heung-Gyoon Ryu [15] planned Orthogonal Frequency Division Multiplexing (OFDM) i.e.., appropriate for top speed communication mistreatment multiple subcarriers. The prevailing OFDM mistreatment an Inverse Fast Fourier Transform (IFFT) uses cyclic prefix to cut back inter-symbol interference. The wave remodel means that the signal is expanded, analysed and rotten by employing a basis operate consistent with completely different wavelets. The simulation was performed by specifying the image amount. The CP utilized in OFDM contains a downside of wasting power and low spectral potency than existing signals. A brand new MCM system is devised to alter high communication by complementing shortcomings of OFDM. Xiaojing Liu et al., [16] planned that the LBP bar graph obtained supported the LBP methodology typically contains a greater length, and not contributory for design. Surface flaw finding is the examination and measuring procedure for areas with non-uniform physical, or chemical possessions on the surface of the article. The Gradient Local Binary Pattern (GLBP) planned during this research could be a flaw finding methodology that mixes the gradient info of the eight-neighbourhood centre purpose. The strategy of this research is especially wont to acquire the location of the subimage wherever the flaw is found, thus on slim dejected the scope for any flaw segmentation. The GLBP methodology supported the sub-image will cut back the info matrix spatial property, and therefore, the quantity of calculation, overcome the effect of noise and different factors. Genevieve Sapijaszko et al., [17] planned DWT execution of the wave remodel that divide a picture into a reciprocally orthogonal set of wavelets. DCT states a finite order of knowledge opinions concerning a total of function periodical at completely dissimilar frequencies. This mixed scheme uses the DCT as a filter that tracks the DWT and is recursively repeated till a threshold is touched. The brink is going to be resolute by the energy amendment of the yield of the DCT and DWT for comparison thereto of the energy of the initial create. The tests used for face documentation scheme mistreatment DWT and DCT in three alternative means by extracting the options of a picture. The scheme utilized an unused method of organization by standardizing the L1-Norm distances to work out an individual's uniqueness.

Huda Mady and Shadi M.S. Hillies [18] planned to improve face recognition methodology supported video transmission underneath light variation, facial expressions, completely dissimilar create, orientation, occlusion, position disparity and motion. Viola-Jones algorithmic rule was used to boost face finding. A mix of Histograms of Orthogonal Gradients (HOG) and LBP were used for face features extraction purpose. Technique applied for face organization were RF and SVM. Menglu Wu and Tongwei Lu [19] have planned a face recognition algorithmic rule supported LBP and native Non-Negative Matrix resolving (LNMF). A constant matrix is obtained through the take a look at sample info that had a projection to non-negative mathematical space. The face image is split into chunks and therefore, the LBP is employed to obtain the block bar graph. Then, the bar graph of the block is connected to the bar graph sequence, that constitutes a brand-new face feature, and therefore, the whole consists of the components. The strategy of the native nonnegative matrix resolving is employed to obtain the nonnegative mathematical space, and therefore, the constant matrix.

\section{PROPOSED MODEL}

In this sector, DWT, OLBP and SVS techniques are used to implement the proposed method for improved face recognition. The technique is examined using regularly available face databases viz., ORL, JAFFE, Yale, and Extended Yale B. The face database images are considered and one level DWT is executed to get the LL sub-band. The LL sub-band is resized and then divided into vertical segments. OLBP is executed on the vertical segments and then vertically flipped. The two types of segments are then 
averaged to get the initial facial features. The averaged initial features are concatenated to obtain concluding features. An image size of $112 \times 92$ considered and used 2D-DWT to develop LL-sub band of size $56 \times 46$. It is then resized to get a size of $56 \times 44$ for SVS. It is divided into four symmetrical vertical segments each of size 56x11. OLBP is applied to each of the vertical segments due to which size reduces to $54 \times 9$ which is then vertically flipped having the same size. Both types of OLBP segments before and after vertical flip are converted to a row vectors of size $486 \times 1$. These segments are then averaged as mentioned in detailed analysis and then concatenated to get the final features having a size of $486 \times 4$ which is converted into a row vector of size $1944 \times 1$. The method is classified into three main sectors viz., Enrolment, Test and Matching Sectors.

\section{A. Enrolment Sector}

The features of face images are extracted in this sector and have a face database, DWT, resize, vertical segments, and concatenation.

\section{- Face Databases:}

(i) ORL Face Database [20]: The database contains four hundred face images taken from forty individuals under numerous lighting conditions, diverse poses, and facial expressions. Each image size is $112 \times 92$, with 256 grey levels per pixel and PGM format.

(ii)Yale Face Database [21]: The database contains one hundred sixty-five faces images of fifteen individuals in GIF format with a size of $320 \times 243$. Each individual has eleven images with varied facial expressions and formations like normal, with glasses, without glasses, right-light, unhappy, sleepy, surprised and wink.

(iii) Japanese Female Facial Expression (JAFFE) [22]: The database consists of two hundred thirteen images of 2223 facial expressions with 22 basic facial expressions and one neutral, modelled by ten Japanese female models. The resolution of the image is $256 \times 256$ with TIFF format.

(iv)Extended Yale B Face database [23]: The database comprises 5760 lone light source images of ten persons each seen under 576 seeing situations of nine postures with sixtyfour light situations. The each of image size is $192 \times 168$ with PGM format.

\section{- Discrete Wavelet Transform (DWT):}

The DWT denotes in terms of mother wavelets and are having a fixed duration of time and frequency, hence signifies in together spatial and frequency domains. It bifurcates into low and high frequency bands as an approximation and detailed bands respectively. The Haar wavelet transform is used in the model which involves averaging and differencing of pixel intensity values. The 2D-DWT computes four bands referred to as LL, LH, HL, and $\mathrm{HH}$ respectively [24]. It is noticed that the LL band has a most essential info band and all other bands have less information. The original image of dimension $112 \times 92$ reduces to $56 \times 46$ in each sub-bands after applying DWT. The proposed research considers only LL band of the DWT and the Fig 1 shows the decomposition of DWT.
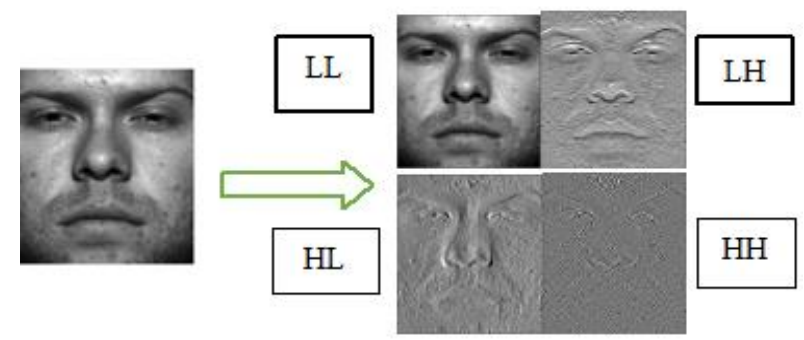

(a) Original Image

(b) DWT Decomposition

Fig 1: - 2D-DWT Decomposition

- Resize of LL sub-band for symmetrical vertical segments:

The LL Sub-band face image is resized in such a way that it can be divided into symmetrical vertical segments. This can be achieved by using the following steps: -

$>$ The rows(r) and columns(c) of the matrix representation of the face image are noted.

$>$ The number of vertical segments to be desired is represented by $2 \mathrm{~S}$ ( $\mathrm{S}$ number of segments in both the left and right side of the face image)

$>$ The generalised formula is applied to obtain the new number of columns represented by $\mathrm{C} 1$

$$
\mathrm{C} 1=\mathrm{c}-(\mathrm{c} \% 2 \mathrm{~S})
$$

where $\mathrm{c} \% 2 \mathrm{~S}$ gives the remainder when $\mathrm{c}$ is divided by $2 \mathrm{~S}$

$>$ The resized image matrix representation with unchanged rows $r$ and changed columns $\mathrm{c} 1$.

\section{Example:}

The size of LL sub band image is $56 \times 46$ i.e., $r=56$ and $c=$ 46 , and the desired $2 \mathrm{~S}$ is 4 ie., two segments left side and two segments right side of an image, then the changed column $\mathrm{C} 1$ is

$$
\begin{gathered}
\mathrm{C} 1=46-(46 \% 4)=46-(2)=44 \\
\text { The resized image will be } 56 \times 44
\end{gathered}
$$

\section{- Symmetrical Vertical Segmentation Method (SVSM)}

The frontal face images are approximately vertically symmetrical in nature around the centre of the face ie., at the nose tip, hence the symmetrical vertical segmentation method [10] is used to extract features from LL sub band image of DWT. The resized LL sub band face image of the size 56x44 is divided into two equal vertical parts results in size of $56 \times 22$ on the left and right side of an image. Further left and right sides of an image are segmented vertically into two equal parts results in four segments with each segment of size 56x11. The resized LL sub band image is vertically segmented into four equal parts as shown in Fig. 2 ie., two left side parts and two right side symmetrical parts.
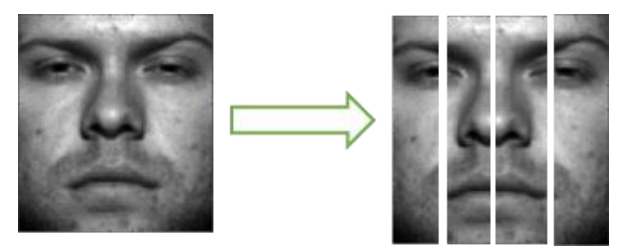

(a) LL sub band Image (b) Vertical Symmetrical Segments

Fig 2: Symmetrical vertical segmentation of resized image 


\section{- Overlapping Local Binary Pattern (OLBP)}

The method is used on each egment of size $56 \times 11$ to extract OLBP features. The method is robust to monotonic greyscale changes due to illumination changes. The LBP operator is invented by Ojala et al., [18] in 1996 by considering a $3 \times 3$ matrix in an image. The centre pixel intensity value $\mathrm{Pc}$ is considered as reference, and the neighbourhood pixel intensity (Pni) values are compared with centre pixel intensity value and convert into binary value (Bni) either 0 or 1 based on equation 1 .

$$
\mathrm{Bni}=\left\{\begin{array}{l}
1, \mathrm{Pni}>\mathrm{Pc} \\
0, \mathrm{Pni}<\mathrm{Pc}
\end{array}\right.
$$

where $n=1$ to 8

The eight binary values are transformed into decimal value and is allocated to centre pixel by replacing original pixel value. This process is continued to cover all pixels in an image by considering overlap $3 \times 3$ except border pixels. The illustration of OLBP is shown in Fig 3. The matrix of size $3 x$ 5 is considered and has three overlapping $3 \times 3$ matrices that are marked to replace centre pixel intensity values by new LBP values. The centre pixel intensity of the first $3 \times 3$ matrix is 55 , and neighbourhood pixel intensity values are replaced by binary based on centre pixel value. The binary value is 10001011 and the corresponding decimal equivalent is 139 . Similarly, centre pixel values 68 and 96 are also replaced by new decimal values of 11 and zero based on LBP operator. It is noticed that the centre pixel values are assigned with new values based on surrounded pixel intensity values.

\begin{tabular}{|c|c|c|c|c|}
\hline 65 & 21 & 3 & 26 & 26 \\
\hline 36 & 55 & 68 & 96 & 26 \\
\hline 25 & 65 & 84 & 77 & 74 \\
\hline
\end{tabular}

\begin{tabular}{|c|c|c|}
\hline 65 & 21 & 3 \\
\hline 36 & 55 & 68 \\
\hline 25 & 65 & 84 \\
\hline
\end{tabular}

(a)3 overlapping $3 \times 3$ blocks

\begin{tabular}{|c|c|c|}
\hline 21 & 3 & 26 \\
\hline 55 & 68 & 96 \\
\hline 65 & 84 & 77 \\
\hline
\end{tabular}

(c) $2^{\text {nd }}$ overlapping $3 \times 3$ block (b) First overlapping $3 \times 3$ block

\begin{tabular}{|l|l|l|}
\hline 3 & 26 & 26 \\
\hline 68 & 96 & 26 \\
\hline 84 & 77 & 74 \\
\hline
\end{tabular}

(d) $3^{\text {rd }}$ overlapping $3 \times 3$ block
The OLBP is applied to each segmented image of size 56 $\mathrm{x} 11$, which resulted in the size of $54 \times 9$. Figure 4 displays a vertical segmented image and the equivalent OLBP image. The OLBP of vertical segments having four segments viz., OLBP of Vertical Segment 1 (OVS1), OVS2, OVS3, and OVS4.

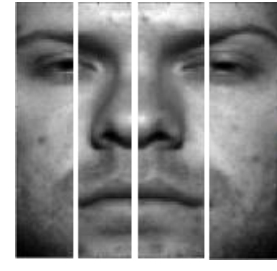

(a)Symmetrical VS
(b)OLBP of Vertical Segments

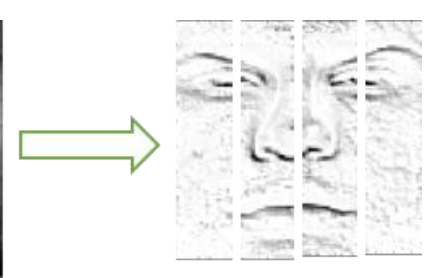

Fig 4: OLBP of four Vertical Segments

- Vertical Flip of OLBP segmented regions:

The OLBP segmented matrix of size $56 \times 11$ is flipped vertically to extract initial features and is demonstrated as follows, consider a matrix $A$ of size mxn

$$
\mathrm{A}=\left[\begin{array}{ccccc}
a 11, & a 12, & a 13, & \cdots & a 1 n \\
a 21, & a 22, & a 23, & \cdots & a 2 n \\
\vdots & \vdots & \vdots & & \vdots \\
a m 1, & a m 2, & a m 3, & \cdots & a m n
\end{array}\right]
$$

$\mathrm{m} \times \mathrm{n}$

Vertical Flip of $A$ is derived from flipped $\mathrm{n} \times \mathrm{n}$ order unit matrix $\mathbf{J}_{\mathrm{nxn}}$

$$
\text { Unit Matrix } I_{n \times n}=\left[\begin{array}{ccccc}
1, & 0, & 0, & \cdots & 0 \\
0, & 1, & 0, & \cdots & 0 \\
0, & 0, & 1, & \cdots & 0 \\
\vdots & \vdots & \vdots & & \vdots \\
0, & 0, & 0, & \cdots & 1
\end{array}\right]
$$

$\mathrm{n} \times \mathrm{n}$

Flipped version of $I_{n \times n}$ is $J_{n \times n}$

$$
\mathrm{J}_{\mathrm{n} \times \mathrm{n}}=\left[\begin{array}{ccccccc}
0, & 0, & 0, & \cdots & 0, & 0, & 1 \\
0, & 0, & 0, & \cdots & 0, & 1, & 0 \\
0, & 0, & 0, & \cdots & 1, & 0, & 0 \\
\vdots & \vdots & \vdots & & \vdots & \vdots & \vdots \\
1, & 0, & 0, & \cdots & 0, & 0, & 0
\end{array}\right]
$$

\section{$\mathrm{n} \times \mathrm{n}$}

Flip matrix of $A^{p}$ is obtained by matrix $A$ multiplied with flipped Identity Matrix $\mathbf{J}_{\mathrm{n} \times \mathrm{n}}$

$$
\begin{aligned}
\mathrm{A}^{\mathrm{p}}=\mathrm{A} . \mathrm{J}_{\mathrm{n} \times \mathrm{n}} & =\left[\begin{array}{cccc}
a 11, & a 12, & \cdots & a 1 n \\
a 21, & a 22, & \cdots & a 2 n \\
a 31, & a 32, & \cdots & a 3 n \\
\vdots & \vdots & & \vdots \\
a m 1, & a m 2, & \cdots & a m n
\end{array}\right]\left[\begin{array}{cccccc}
0, & 0, & \cdots & 0, & 0, & 1 \\
0, & 0, & \cdots & 0, & 1, & 0 \\
0, & 0, & \cdots & 1, & 0, & 0 \\
\vdots & \vdots & & \vdots & \vdots & \vdots \\
1, & 0, & \cdots & 0, & 0, & 0
\end{array}\right] \\
& =\left[\begin{array}{ccc}
a 1 n, & \cdots & a 11 \\
a 2 n, & \cdots & a 21 \\
a 3 n, & \cdots & a 31 \\
\vdots & & \vdots \\
a m n, & \cdots & a m 1
\end{array}\right]
\end{aligned}
$$


Example:

$$
\text { If } \begin{aligned}
A & =\left[\begin{array}{cccc}
1 & 2 & 3 & 4 \\
5 & 6 & 7 & 8 \\
9 & 10 & 11 & 12
\end{array}\right] \\
I & =\left[\begin{array}{llll}
1 & 0 & 0 & 0 \\
0 & 1 & 0 & 0 \\
0 & 0 & 1 & 0 \\
0 & 0 & 0 & 1
\end{array}\right]{ }_{4 \times 4}
\end{aligned}
$$

Flip of $I$ is $J$

$$
J=\left[\begin{array}{llll}
0 & 0 & 0 & 1 \\
0 & 0 & 1 & 0 \\
0 & 1 & 0 & 0 \\
1 & 0 & 0 & 0
\end{array}\right]
$$

The Flip of $\mathrm{A}$ is $\mathrm{A}^{\mathrm{p}}=\mathrm{A}$. $\mathrm{J}$

$$
\begin{aligned}
& =\left[\begin{array}{cccc}
1 & 2 & 3 & 4 \\
5 & 6 & 7 & 8 \\
9 & 10 & 11 & 12
\end{array}\right]\left[\begin{array}{llll}
0 & 0 & 0 & 1 \\
0 & 0 & 1 & 0 \\
0 & 1 & 0 & 0 \\
1 & 0 & 0 & 0
\end{array}\right] \\
& =\left[\begin{array}{cccc}
4 & 3 & 2 & 1 \\
8 & 7 & 6 & 5 \\
12 & 11 & 10 & 9
\end{array}\right]
\end{aligned}
$$$$
3 \times 4
$$

The four OLBP segmented images are flipped and are shown in Figure 5 as Flipped OLBP Vertical Segments FOVS 1, FOVS2, FOVS3, and FOVS4.

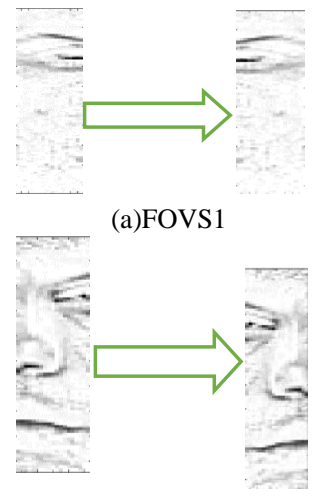

(c)FOVS3

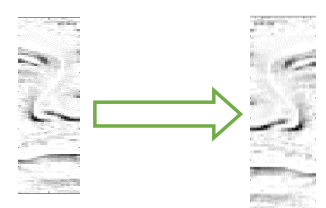

(b) FOVS2

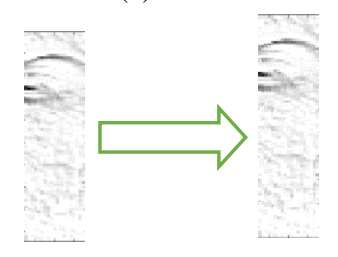

(d) FOVS4
Fig 5: Vertical Flip of OLBP segmented regions

- Average of OLBP and OLBP flip segments:

The OLBP features of vertical segment and flipped OLBP vertical segment features are combined to obtain Intermediate Features (IF) by averaging segment wise as in equations 2 - 5 .

$$
\begin{aligned}
& I F 1=\frac{\text { ovs } 1+F \text { OVS } 4}{2} \\
& I F 2=\frac{\text { ovs } 2+\text { Fovs } 3}{2} \\
& I F 3=\frac{\text { ovs } 3+\text { Fovs } 2}{2} \\
& I F 4=\frac{\text { ovs } 4+\text { Fovs } 1}{2}
\end{aligned}
$$

The four IF's are obtained by combination of average of OLBP and Flipped OLBP segments is as shown in Fig 6.

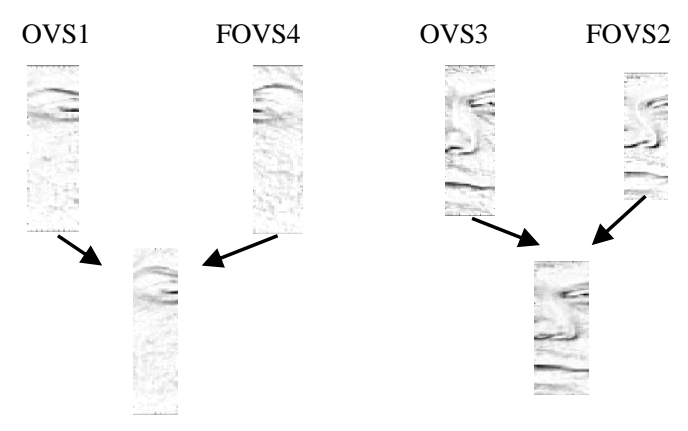

Fig 6: Average images

\section{- Final Feature (FF)}

The average features of all four segments are considered and concatenated to obtain final features using an expression 6. The final image after processing of DWT, segments, OLBP's and Flipped OLBP's is as shown in Fig 7

$$
\mathrm{FF}=[\mathrm{IF} 1 ; \mathrm{IF} 2 ; \mathrm{IF} 3 ; \mathrm{IF} 4]
$$

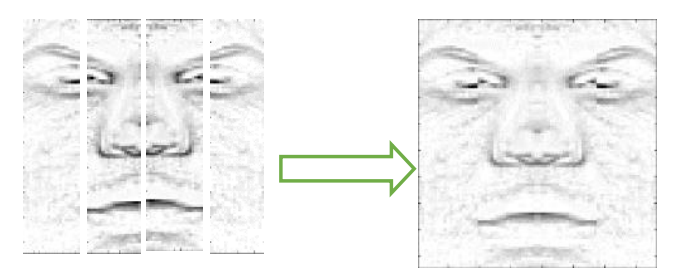
(a)Average
(b) Concatenated final image

Fig 7: - Final Averaged Segments

B. Test Sector:

The face images to be tested are used in this section and the procedure to extract final features based on DWT, segmentation, flipping, and OLBP is the same as that of the enrolment sector.

\section{Matching Sector:}

The query image features are equated to the database image features by ED formula as given in Equation 7.

$$
D(p, q)=\sqrt{\frac{1}{M} \sum_{i=1}^{M}\left(p_{i}-q_{i}\right)^{2}}
$$


Input: Face image databases, probe face images.

Output: Computation of performance constraints.

Step 1: Face images are loaded from the database and RGB images are converted to greyscale images.

Step 2: Single Level Two Dimensional DWT is used on face images and considered the LL band, which is resized.

Step 3: The LL sub-band is resized and decomposed into four vertical segments.

Step 4: OLBP is applied to all the vertical segments.

Step 5: The four OLBP segments are Vertically Flipped

Step 6: Averaging of segments obtained in step 4 and

Step 7: Concatenation of segments obtained in step 6.

Step 8: Repeat steps 1 to 7 for Test Images also.

Step 9: Match the features of face database images and probe images using ED to calculate output results.

$\mathrm{M}=$ the feature vector dimension

$\mathrm{p}_{\mathrm{i}}=$ database features

$\mathrm{q}_{\mathrm{i}}=$ test features

\section{ILLUSTRATION OF PROPOSED FEATURE EXTRACTION MODEL}

The original image of size $112 \times 92$ is considered, and extracted final features based on DWT, Segmentation and OLBP is as shown in Fig 8. The DWT is used on the original image and only the LL band is considered, which is a compressed version and free from the noise of size $56 \times 46$. The LL band is resized to $56 \times 44$ for the purpose of four vertical segments (VS1), VS2, VS3 and VS4 of each of size $56 \times 11$. The OLBP is applied to each segment to obtain OLBP Vertical Segments OSV1, OSV2, OSV3, and OSV4. The process of vertical flip technique is applied on OLBP Vertical Segments to obtain flipped OLBP vertical segment FOSV1, FOSV2, FOSV3, and FOSV4. The average of OLBP segments and flipped OLBP vertical segments are computed for intermediate features. The final effective features are obtained by contacting all four intermediate features.

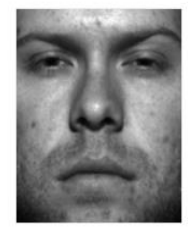

(a) Original Image (112x92) OSV1 OSV2 OSV3 OSV4

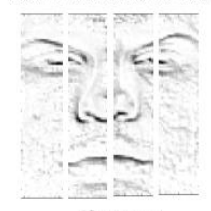

(f) OLBP $\square$

FOSV1 FOSV2 FOSV3 FOSV4

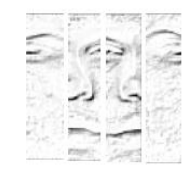

(g)Vertical Flip

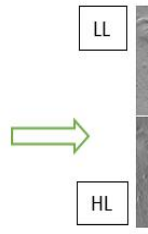

(b) 1 LEVEL 2D DWT (c) LL Band (56x46)
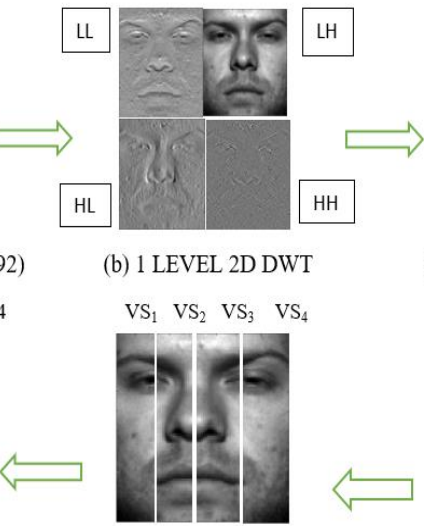

$\begin{array}{llll}\mathrm{VS}_{1} & \mathrm{VS}_{2} & \mathrm{VS}_{3} & \mathrm{VS}_{4}\end{array}$

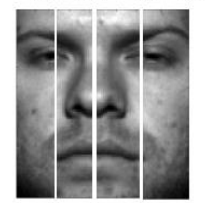

(e)Vertical Segments (56x11 each)
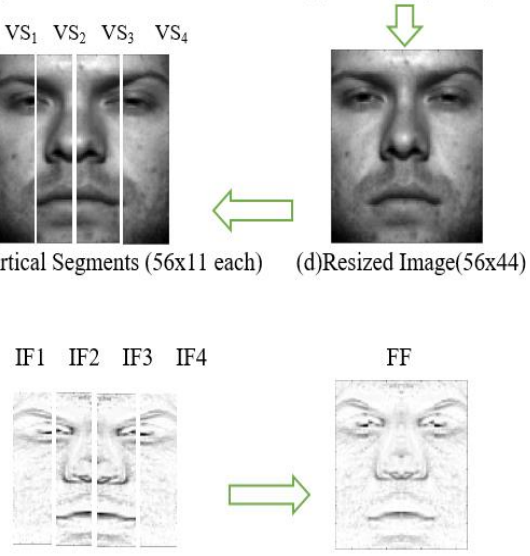

(h)Average
$\mathrm{FF}$

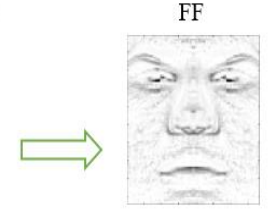

(i)Concatenated Image
Fig 8: - The illustration of the proposed Feature Extraction process

\section{PROPOSED ALGORITHM}

\section{- Problem Definition:}

The proposed algorithm is developed for human face recognition which has high recognition and fewer error rates. The algorithm is robust in nature that is not affected by illumination, pose and expression variation as well as occlusion, aging and disguise are set in Table 1.

\section{- Objectives:}

(i)Recognition of an individual using face images.

(ii)To attain higher TSR values.

(iii)To attain low EER values.

\section{TABLE 1: PROPOSED ALGORITHM}

\section{EXPERIMENTAL RESULTS AND INVESTIGATION}

In this sector, the definitions of performance constraints, experimental results, and investigations using numerous face databases are deliberated. The performance constraints are calculated for dissimilar mixtures of PID and PODs [23].

\section{A. Definitions}

- False Accept Rate (FAR): Images in the test section from the outside database are match with images in the enrolment section. The FAR is calculated using equation 12

FAR $=\frac{\text { Total number of persons incorrectly accepted in enrolment section from out of database }}{\text { Total number of persons in enrolment section }}$

- False Rejection Rate (FRR): Images in the test section from the inside database are mismatches with images in the enrolment section and is computed using equation 13

$$
\text { FRR }=\frac{\text { No.of genuine persons in the test section are rejected }}{\text { Total no. of persons in enrolment section }}
$$

- Equal Error Rate (EER): The optimum error value for which the FRR and FAR values are minimum and equal at the optimum threshold value.

- True Success Rate (TSR): The whole number of people in the test section are appropriately matched with the number of individuals in the database and is specified by equation 14

$$
\mathrm{TSR}=\frac{\text { Total number of persons correctly Matched in the database }}{\text { Total number of persons in database }}
$$

- Optimum True Success Rate (OTSR): The value of TSR at point of EER.

- Maximum True Success Rate (MTSR): Maximum value of TSR

\section{B. Performance Evaluation:}

The proposed method is evaluated using ORL, JAFFE, Extended Yale B, and Yale 
- Result investigations via ORL face database:

The performance constraints viz., \%FRR, \%FAR and \% TSR are calculated aimed at variants in threshold values and the plots are found as revealed in Figures 9 to 11 meant for PID and POD combinations of 10:20, 10:30, and 20:20. The experimental values of FRR falls through a rise in threshold values while, the FAR and TSR values rises by values of threshold. The corresponding values of EER used for PID and POD mixtures of 10:20, 10:30, and 20:20 are $27 \%, 28 \%$, and $30 \%$.

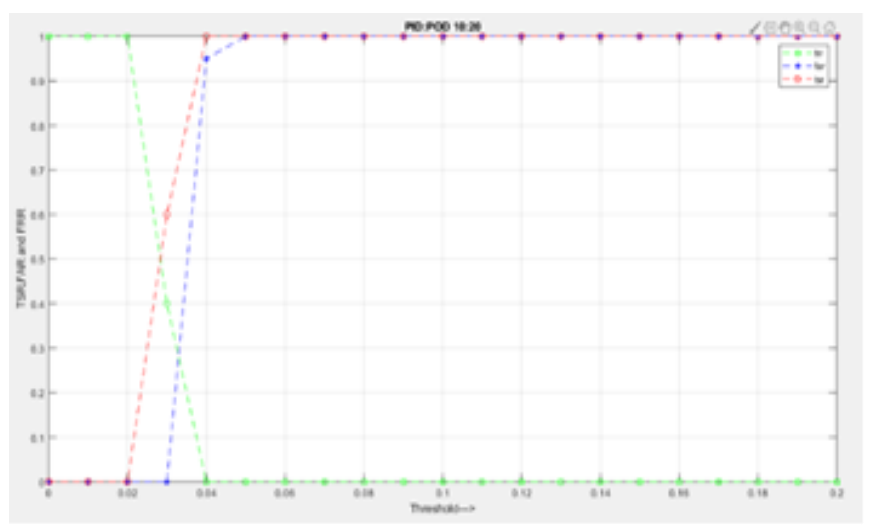

Fig 9: Performance constraints with threshold aimed at PID and POD combination of 10:20

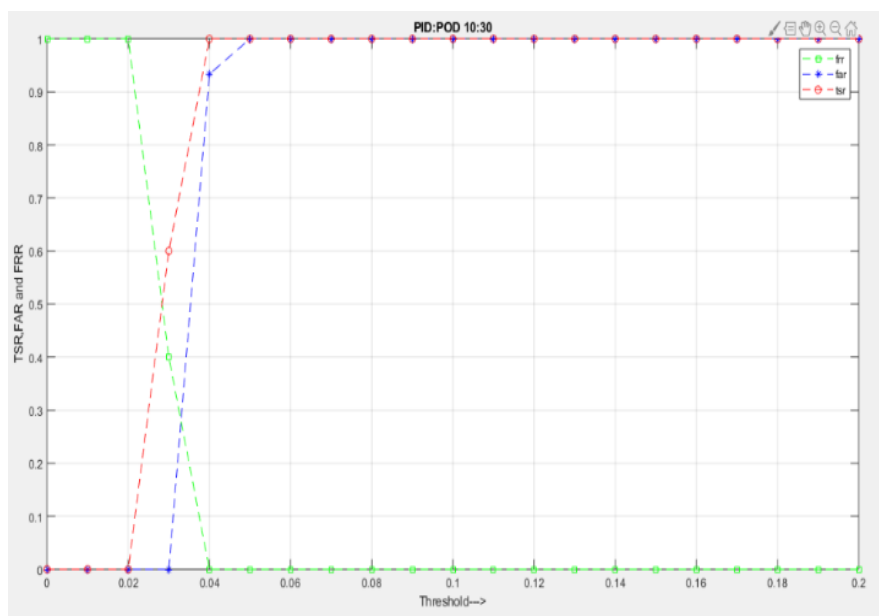

Fig 10: Performance constraints with threshold aimed at PID and POD mixture of 10:30

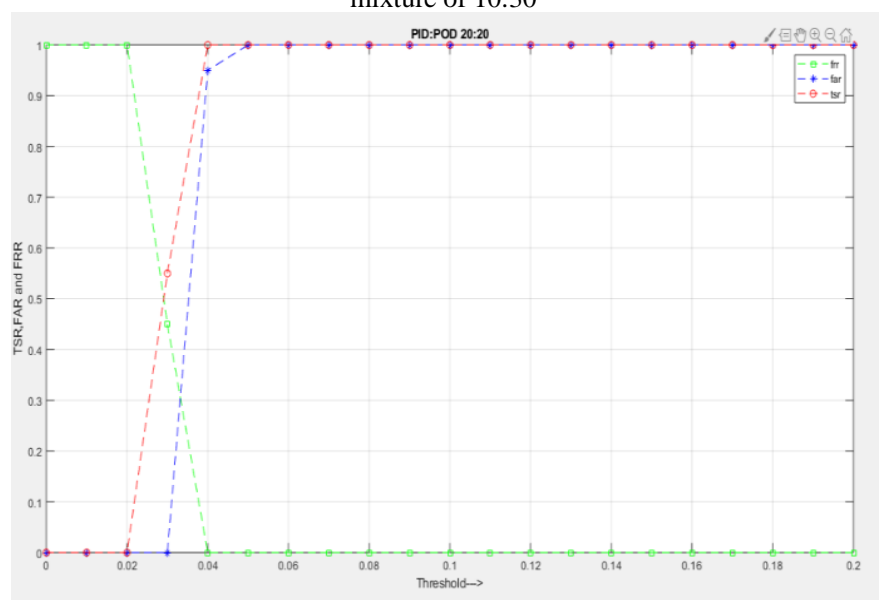

Fig 11: - Performance constraints with threshold aimed at PID and POD mixture of 20:20
The deviations of \%EER, \%OTSR, and \%MTSR for different PID and POD mixtures are presented in Table 2. It is noticed that the error rises with rise in PID and POD values. The percentage of OTSR values drops, whereas MTSR remains constant at $100 \%$ for rise in values of PID's.

TABLE 2: - DEVIATIONS IN CONSTRAINTS THROUGH ORL FACE

\begin{tabular}{|c|c|c|c|c|c|}
\hline $\begin{array}{l}\text { SL } \\
\text { NO. }\end{array}$ & PID & POD & \%EER & \%OTSR & \% MTSR \\
\hline 1 & 10 & 20 & 27 & 72 & 100 \\
\hline 2 & 10 & 30 & 28 & 71 & 100 \\
\hline 3 & 20 & 20 & 30 & 70 & 100 \\
\hline
\end{tabular}

- Result Investigations via JAFFE Face Database:

The performance constraints viz., \%FRR, \%FAR and \% TSR are calculated aimed at variants in values of threshold and the plots are found as revealed in Figures 12 to 14 aimed at PID and POD mixtures of 3:5, 3:7, and 5:5 respectively. The experimental values of \%FRR drops through rise in values of threshold while, the values of \%FAR and \%TSR rises through threshold values. The corresponding \%EER values for PID and POD mixtures of 3:5, 3:7, and 5:5 are $32 \%, 35 \%$, and $36 \%$ respectively.

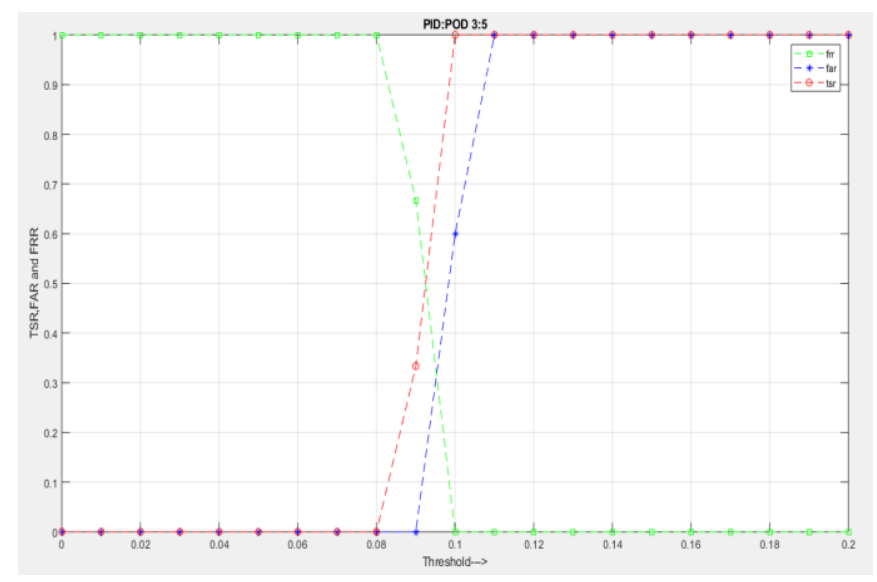

Fig 12: Performance constraints with threshold aimed at PID and POD combination of $3: 5$

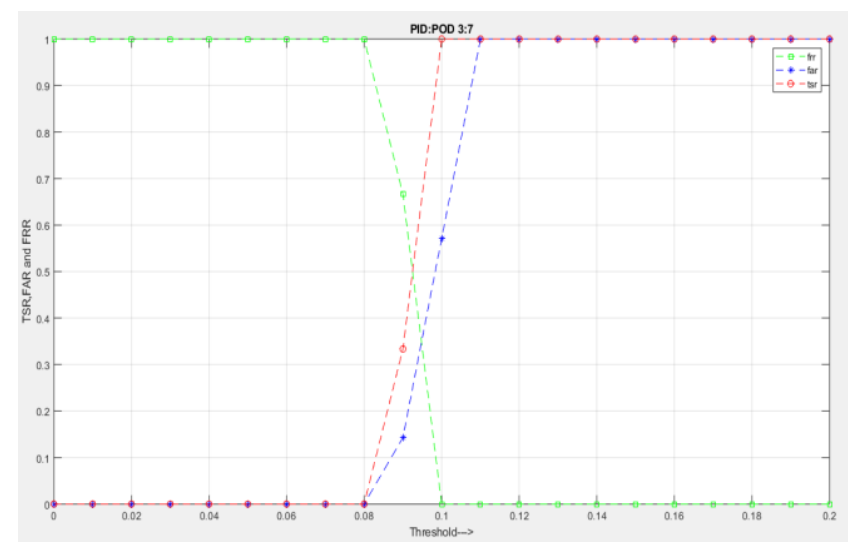

Fig 13: Performance constraints with threshold aimed at PID and POD combination of $3: 7$ 


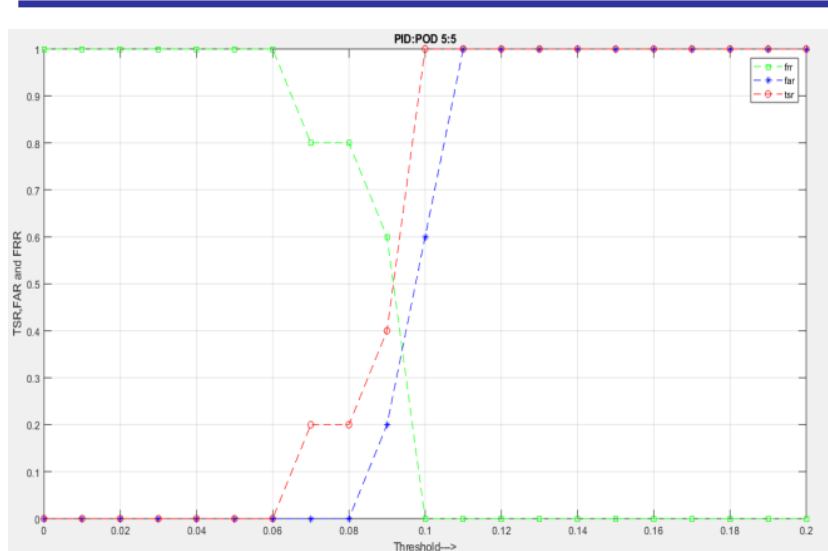

Fig 14: Performance constraints with threshold aimed at PID and POD combination of 5:5

The deviations of \%EER, \%OTSR and\% MTSR for different PID and POD mixtures are presented in Table 3. It is noticed that the error rises and \% OTSR falls with a rise in the values of PID and POD's.

TABLE 3: VARIATIONS IN CONSTRAINTS WITH JAFFE FACE

\begin{tabular}{|c|c|c|c|c|c|}
\hline $\begin{array}{c}\text { SL } \\
\text { NO. }\end{array}$ & PID & POD & \%EER & \%OTSR & \%MTSR \\
\hline 1 & 3 & 5 & 32 & 68 & 100 \\
\hline 2 & 3 & 7 & 35 & 65 & 100 \\
\hline 3 & 5 & 5 & 36 & 64 & 100 \\
\hline
\end{tabular}

- Result investigations using Extended Yale B Face Database: -

The performance constraints viz., \%FRR, \%FAR, and \%TSR are calculated for variants in values of threshold and the plots are found as revealed in Figures 15 to 17 for PID and POD combinations of 10:20, 18:18 and 20:10 respectively. The experimental values of \%FRR fall by the rise in values of threshold while the values of $\%$ FAR and $\%$ TSR rises through threshold values. The corresponding $\%$ EER value is $0 \%$ for all mixtures of PID and POD's.

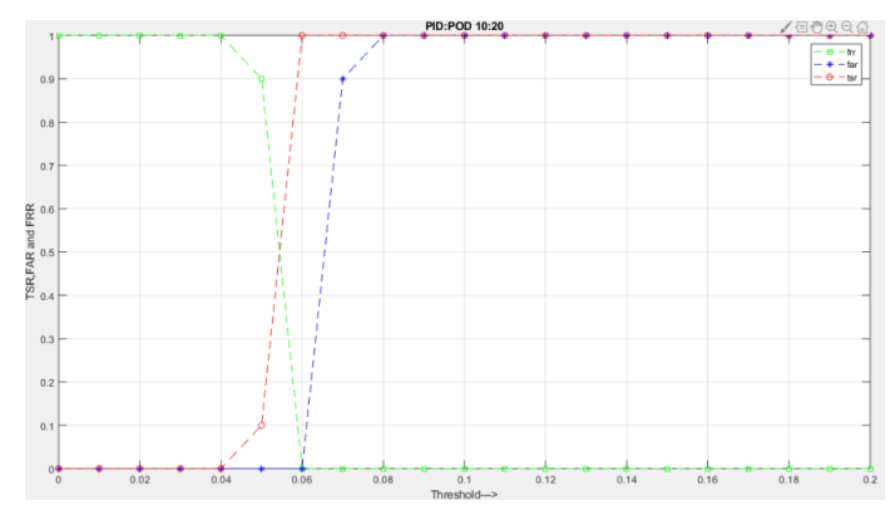

Fig 15: - Performance constraints with threshold aimed at PID and POD combination of 10:20

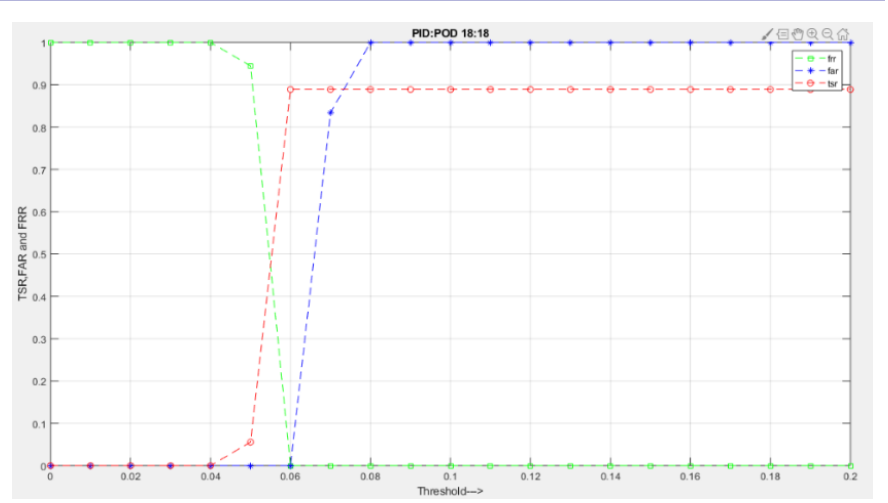

Fig 16: - Performance constraints with threshold aimed at PID and POD combination of $18: 18$

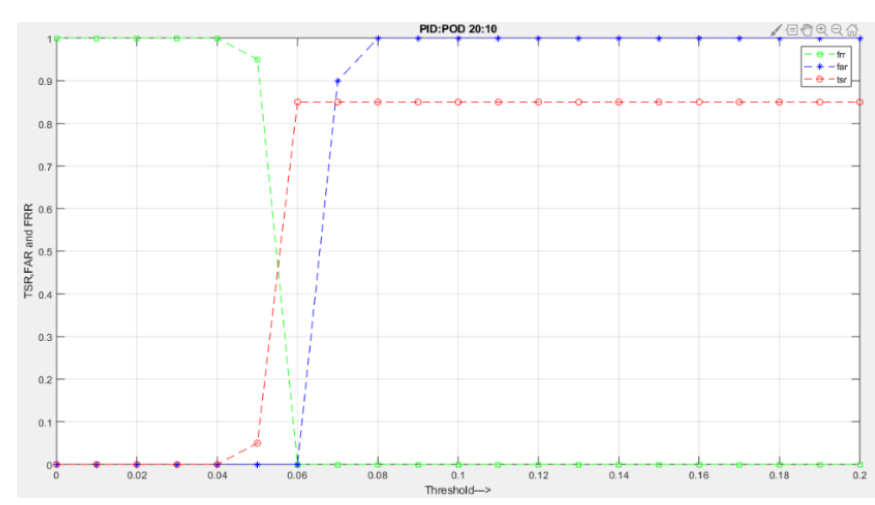

Fig 17: - Performance constraints with threshold aimed at PID and POD combination of $20: 10$

The deviations of \%EER, \%OTSR, and \%MTSR for different PID and POD mixtures are presented in Table 4. It is noticed that the error remains constant at zero and \%OTSR drops with a rise in the values of PID. The maximum \%TSR value is 100 for three combinations of PID and POD's.

TABLE 4: - VARIATIONS OF CONSTRAINTS WITH EXTENDED YALE B FACE DATABASE

\begin{tabular}{|c|c|c|c|c|c|}
\hline $\begin{array}{c}\text { SL } \\
\text { NO. }\end{array}$ & PID & POD & \%EER & \%OTSR & \%MTSR \\
\hline 1 & 10 & 20 & 0 & 100 & 100 \\
\hline 2 & 18 & 18 & 0 & 89 & 100 \\
\hline 3 & 20 & 10 & 0 & 85 & 100 \\
\hline
\end{tabular}

- Result investigations via Yale face database:

The performance constraints viz., \%FRR, \%FAR and $\%$ TSR are calculated aimed at variants in values of threshold and the plots are found as revealed in Figures 18 to 20 for PID and POD mixtures of 7:3, 7:7, and 10:5 respectively. The experimental values of $\%$ FRR falls by rise in threshold values while, the values of \%FAR and \%TSR rises by values of threshold. The corresponding \%EER values aimed at PID and POD mixtures of $7: 3,7: 7$, and $10: 5$ are $40 \%, 45 \%$, and $50 \%$ respectively. 


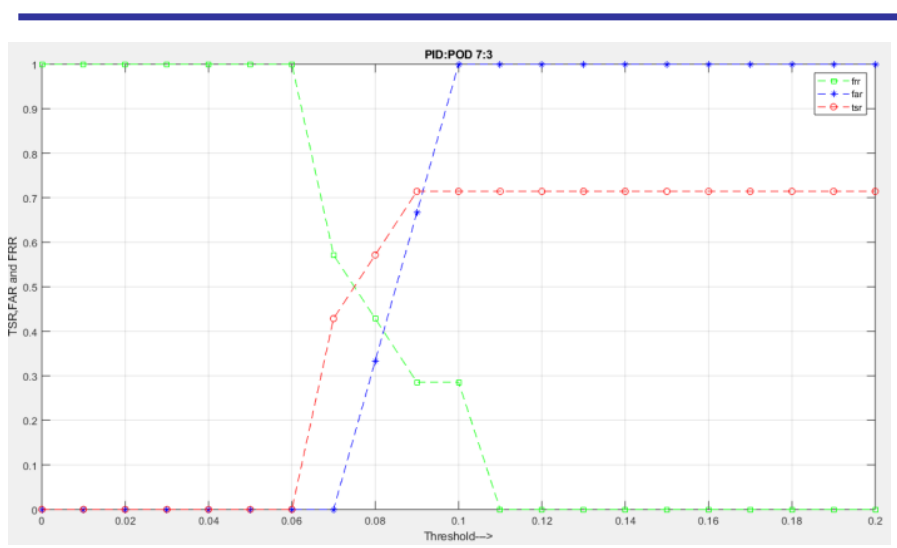

Fig 18: - Performance constraints with threshold aimed at PID and POD combination of 7:3

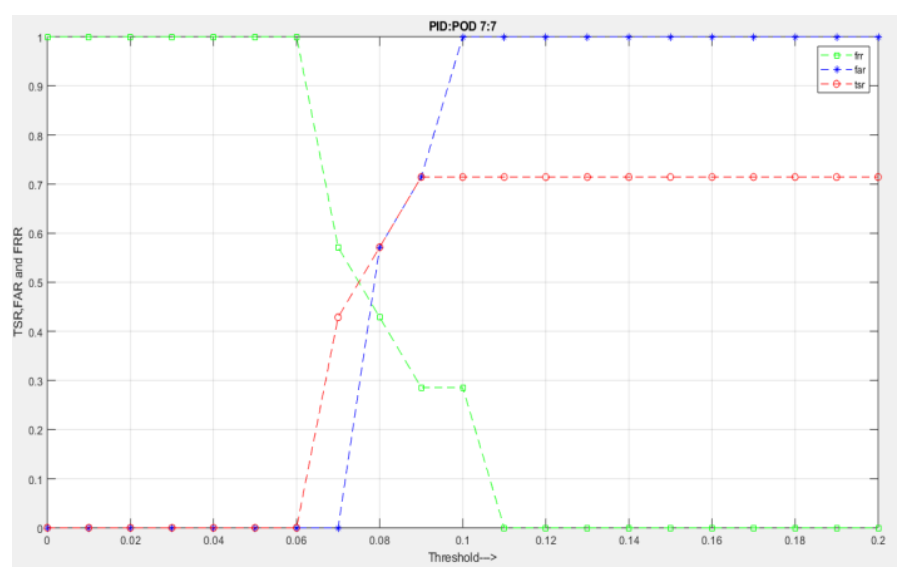

Fig 19: - Performance constraints with threshold aimed at PID and POD combination of $7: 7$

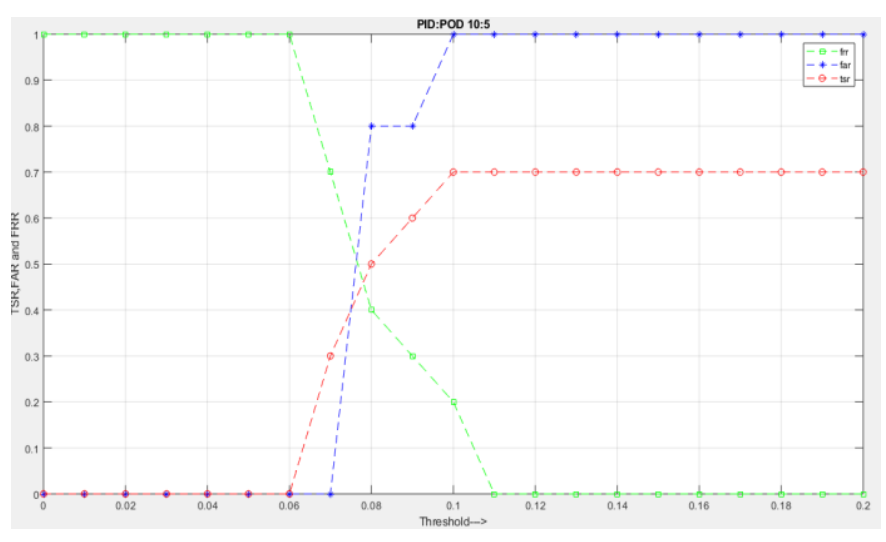

Fig 20: - Performance constraints with threshold aimed at PID and POD combination of 10:5

The deviations of \%EER, \%OTSR, and \%MTSR for different PID and POD mixtures are presented in Table 5. It is detected that the \%OTSR and \%MTSR drops with a rise in PID.
TABLE 5: - VARIATIONS IN PERFORMANCE CONSTRAINTS THROUGH YALE FACE DATABASE

\begin{tabular}{|c|c|c|c|c|c|}
\hline $\begin{array}{c}\text { SL } \\
\text { NO. }\end{array}$ & PID & POD & \%EER & \%OTSR & \%MTSR \\
\hline 1 & 7 & 3 & 40 & 60 & 71.5 \\
\hline 2 & 7 & 7 & 45 & 55 & 71.5 \\
\hline 3 & 10 & 5 & 50 & 42.5 & 70 \\
\hline
\end{tabular}

\section{Comparison of Recognition Rate of Proposed Method} Thru Existing Methods:

The recognition rate of the proposed technique is compared by current techniques offered by Eyad I. Abbas et al., [26] for ORL face database, Shui-Guang Tong et al., [10] for Extended Yale-B face database, and Asit Barman and Paramartha Dutta [27] for JAFFE face databases are quantified in Table 6. The \%MTSR is elevated in the case of the proposed technique related to current techniques.

TABLE 6: - THE \%MTSR OF PROPOSED TECHNIQUE IS COMPARED THROUGH CURRENT TECHNIQUES

\begin{tabular}{|c|c|c|c|}
\hline $\begin{array}{c}\text { SL } \\
\text { NO. }\end{array}$ & FACE & AUTHORS & \% MTSR \\
\hline 1 & ORL & Eyad I. Abbas et al. [26] & 98 \\
\cline { 3 - 4 } & & Proposed Method & 100 \\
\hline 2 & \multirow{2}{*}{ Extended Yale B } & Shui-Guang Tong et al. [10] & 93.8 \\
\cline { 3 - 4 } & & Proposed Method & 100 \\
\hline 3 & \multirow{2}{*}{ JAFFE } & $\begin{array}{c}\text { Asit Barman and Paramartha } \\
\text { Dutta[27] }\end{array}$ & 92.8 \\
\cline { 3 - 4 } & & Proposed Method & 100 \\
\hline
\end{tabular}

\section{CONCLUSION}

The person authentication using face images is a challenging task for natural variations in face images for recent secure applications. OLBP on DWT Segments for Effective Face Recognition is proposed in this research. The LL band is generated by applying DWT on face images and the LL band is resized for four vertical segments. The texture features of the four segments are obtained by the OLBP procedure. The four OLBP segments are flipped and combined with corresponding original OLBP segments to compute average values to obtain initial features. The average OLBP values of four segments are concatenated to derive final effective features. The final features of the enrolled database and test images are related by ED to calculate performance constraints. The proposed method is improved compared to current techniques. In future DWT can be replaced by Dual Tree Complex Wavelet Transform to test the proposed method. 


\section{REFERENCES}

[1] X Geng, Z H Zhou, and K Smith-Miles, "Automatic Age Estimation based on Facial Aging Patterns" IEEE Transactions on Pattern Analysis and Machine Intelligence, vol 29, no 12, pp. 2234-2240, 2007

[2] U. Park, Y. Y. Tong, A. K. Jain. "Age-Invariant Face Recognition", IEEE Transactions on Pattern Analysis and Machine Intelligence, vol. 32, no. 5, pp. 947-954, 2010

[3] X. Geng, C Yin, and Z H Zhou, "Facial Age Estimation by Learning from Label Distributions", IEEE Transactions on Pattern Analysis and Machine Intelligence, vol. 35, no. 10, pp. 2401-2412, 2013.

[4] C X Ren, D Q Dai, and H Yan, "Coupled Kernel Embedding for LowResolution Face Image Recognition", IEEE Transactions on Image Processing, vol. 21, no. 8, pp. 3770-3783, 2012.

[5] WWW. Zou, and P. C. Yuen, "Very Low-Resolution Face Recognition Problem", IEEE Transactions on Image Processing, vol. 21, no. 1, pp. 327-340, 2012

[6] H. Drira, B. Ben Amor, A. Srivastava, M. Daoudi, and R. Slama. "3D Face Recognition Under Expressions, Occlusions, and Pose Variations", IEEE Transactions on Pattern Analysis and Machine Intelligence, vol. 35, no. 9, pp. 2270-2283, 2013

[7] G. Passalis, P. Perakis, T. Theoharis,

I. A. Kakadiaris, "Using facial

symmetry to handle pose variations in real-world 3D face recognition", IEEE Transactions on Pattern Analysis and Machine Intelligence, vol. 33, no. 10, pp. 1938-1951, 2011.

[8] X. Y. Tan, and B. Triggs. "Enhanced Local Texture Feature Sets for Face Recognition Under Difficult Lighting Conditions", IEEE Transactions on Image Processing, vol. 19, no. 6, pp. 1635-1650, 2010.

[9] T. Ojala, M. Pietikäinen, and D. Harwood, "A Comparative Study of Texture Measures with Classification based on Feature Distributions", Pattern Recognition, vol. 29, no. 1, pp. 51-59, 1996.

[10] Shui-Guang Tong, Yuan-Yuan Huang and Zhe-Ming Tong "A Robust Face Recognition Method Combining LBP with Multi-Mirror Symmetry for Images with Various Face Interferences", International Journal of Automation and Computing, Springer, pp. 1-12, 2018.

[11] Bin Xiao, Kaili Wang, Xiuli Bi, Weisheng Li, and Junwei Han "2DLBP: An Enhanced Local Binary Feature for Texture Image assification," IEEE Transactions on Circuits and Systems for Video Technology, vol. 29, NO. 9, pp 2796-2808, September 2019

[12] Chao Qi, Min Li, Qiushi Wang, Huiquan Zhang and Jinling Xing "Facial Expressions Recognition Based on Cognition and Mapped Binary Patterns", IEEE Access Volume 6, pp. 18785-18803 2169-3536, 2018

[13] Latha S and Savita S Kudakunti, "Face Recognition Using Transform Domain and Overlapping LBP Techniques", International Journal of Engineering Research \& Technology, pp. 343-347, 2017
[14] Arti Mahore and Meenakshi Tripathi, "Detection of 3D Mask in 2D Face Recognition System Using DWT and LBP" IEEE 3rd International Conference on Communication and Information Systems, pp. 18-22, 2018

[15] Jun-Gu Lee and Heung-Gyoon Ryu "Design and Comparison of Discrete Wavelet Transform Based OFDM (DWT-OFDM) system" IEEE International Conference on Ubiquitous and Future Networks, pp. 881-885, 2018

[16] Xiaojing Liu, Lu Tent and Feng Xue "Surface Defect Detection Based on Gradient LBP” IEEE International Conference on Image, Vision and Computing, pp. 133-137, 2018

[17] Genevieve Sapijaszko, Taif Alobaidi and Wasfy B. Mikhael "Adaptive Feature Extraction Algorithm using Mixed Transforms for Facial Recognition", IEEE Transactions on Image Processing, Vol. 24, No. 12, pp. $226-229,2018$

[18] Huda Mady and Shadi M.S., "Face Recognition and Detection using Random Forest and Combination of LBP and HOG Features", International Conference on Smart Computing and Electronic Enterprise, pp. 1-17, 2018.

[19] Menglu Wu and Tongwei Lu, "Face Recognition Based on LBP and LNMF Algorithm", International Symposium on Parallel and Distributed Computing, pp. 368-371, 2016

[20] ORL Database, http://www.camrol.co.uk

[21] http://www.yaledatabase.com

[22] http://www.kasrl.org/jaffe_download.html

[23] http://vision.ucsd.edu/ iskwak/ExtYaleDatabase/ExtYaleB.htm

[24] Sujatha B M, Chetan Tippanna Madiwalar, K Suresh Babu, K B Raja and Venugopal K R "Compression Based Face Recognition Using DWT and SVM" International Journal of Signal \& Image Processing(SIPIJ), Vol 7, no.3, pp 45-62, June 2016

[25] Ganapathi V Sagar, Savita Y Barker, K B Raja, K Suresh Babu and K R Venugopal " A Feature Vector Compression Approach for Face Recognition using Convolution and DWT", International Journal of Computer and Technology, vol.15, no.1, pp.6453 - 6470, November, 2015.

[26] Eyad I. Abbas, Mohammed E. Saf and Khalida S. Rijab, "Face Recognition Rate Using Different Classifier Methods Based on PCA", IEEE International Conference on Current Research in Computer Science and Information Technology (ICCIT), pp. 37-40, 2017

[27] Asit Barman and Paramartha Dutta "Texture Signature based Facial Expression Recognition using NARX”, IEEE Calcutta Conference (CALCON), pp. 6-10, 2017 